\title{
Coagulation and Aging: Implications for the Anesthesiologist
}

\author{
Sarina L. $\operatorname{Tschan}^{1} \cdot$ Daniel Bolliger ${ }^{2} \mathbb{C}$ \\ Accepted: 18 October 2021 / Published online: 19 November 2021 \\ (c) The Author(s) 2021
}

\begin{abstract}
Purpose of Review This narrative review focuses on aging-related modifications in coagulation resulting in increased thromboembolic and hemorrhagic risk of the elderly. We further discuss the current evidence and emerging data relating the perioperative treatment of elderly patients with antithrombotic therapy.

Recent Findings Relevant changes in all elements of the Virchow's triad can be found with aging. Increased blood stasis due to immobility, progressive endothelial dysfunction with altered microcirculation, elevated concentrations of several coagulation factors, and increased platelet reactivity all lead to a procoagulant state. Elderly people are, therefore, commonly treated with oral anticoagulation and antiplatelet drugs. This antithrombotic therapy might be essentially causative for their increased bleeding risk.

Summary Elderly patients are at increased risk for thromboembolism due to changes in the hemostatic system in combination with frailty and multimorbidity. Both the thromboembolic due to aging and bleeding risk due to antithrombotic therapy need special attention in the elderly surgical patients.
\end{abstract}

Keywords Aging $\cdot$ Coagulation $\cdot$ Perioperative management $\cdot$ Thromboembolism

\section{Introduction}

According to the United Nations' World Population Prospects 2019, more than 700 million persons worldwide are older than 65 years of age [1]. This number is expected to double to about 1.5 billion elderly people in 2050 due to advances in science and medicine, meaning that one in six people will be aged $>65$ years. Despite the more recent definition of "elderly" currently applied to those aged $>75$ years, the increasing elderly population represents a major social,

This article is part of the Topical Collection on Geriatric Anesthesia

Daniel Bolliger

daniel.bolliger@usb.ch

Sarina L. Tschan

sarinalaura.tschan@usb.ch

1 Clinic for Anaesthesiology, Intermediate Care, Prehospital Emergency Medicine, and Pain Therapy, University Basel Hospital, Spitalstrass 21, CH-4031 Basel, Switzerland

2 Clinic for Anaesthesiology, Intermediate Care, Prehospital Emergency Medicine, and Pain Therapy, University Basel Hospital, University of Basel, Spitalstrasse 21, CH-4031 Basel, Switzerland economic, and medical challenge. Of note, the number of elderly patients presenting for surgical interventions will further increase [2]. For the perioperative physician, physical deterioration associated with frailty, multiple comorbidities, and polypharmacy are distinctive features [ $3 \bullet$ rendering these patients highly vulnerable in the perioperative period.

Under physiologic conditions, a hemostatic balance is achieved by complex interactions occurring between plasma coagulation proteins and enzymes, platelets, and other cellular elements including the vessel wall, eventually protecting the body from bleeding and inadvertent clot formation. Changes associated with aging might result in shifting this delicate balance towards thrombosis and bleeding disorders. In general, an increased risk of arterial and venous thrombosis has been associated with aging $[4,56,7 \bullet]$. Due to the increased thromboembolic risk and comorbidities, elderly patients are more commonly treated with anticoagulants and antiplatelet drugs than younger patients. Therefore, elderly patients frequently present with bleeding complications. Both the increased perioperative thromboembolic risk due to procoagulant changes associated with aging and the bleeding risk due to therapeutic interventions against thromboembolism need special attention in elderly patients scheduled for surgical procedures. 
This review will focus on the aging-related hemostatic changes of coagulation and discuss the comorbidities contributing to an increased risk of thrombosis and bleeding events in the elderly patients. In addition, the current evidence and emerging data relating to the perioperative treatment of elderly patients with anticoagulants are discussed.

\section{Search Strategy}

An extensive literature search in PubMed was performed using the following terms: (coagulation) AND (aging); and (coagulation) AND (elderly) AND (perioperative). The search was restricted to clinical studies, clinical trials, metaanalysis, randomized controlled trials (RCT), reviews, and systematic reviews. Only publications in English between January 2000 and March 2021 were included, resulting in the identification of 526 and 611 references, respectively. Publications with potential importance were critically reviewed and eventually included in this publication.

\section{Hemostatic Changes in the Elderly}

Hemostasis involves a complex series of procoagulant and fibrinolytic processes controlled by inhibitors and feedback mechanisms [8]. With aging, alterations in the hemostatic balance lead to a tendency towards thrombophilia and an increased risk for thromboembolic events and complications. For many years, the pathogenesis of thromboembolism has focused on the classical Virchow's triad including blood stasis, changes of the endothelium and the vessel walls, and hypercoagulability. Recently, vessel wall alteration, systemic inflammation, and infections with consecutive activation of the coagulation system have been suggested to be the main drivers of thromboembolism. In contrast, stasis might primarily act as a permissive condition rather than as a direct cause. However, relevant changes in all elements of Virchow's triad can be found with aging. First, elderly people are generally frail, have sedentary lifestyles, suffer from diseases that restrict mobility, and might require surgical interventions more often than younger people. All of these factors contribute to immobility and increased blood stasis. Second, aging of the vascular system leads to changes in vessel wall integrity and microcirculation [6]. The latter is aggravated by progressive endothelial dysfunction associated with aging. Such endothelial dysfunction leads to increased vascular permeability, reduced vascular tone, and loss of the endothelium's ability to regulate and control hemostatic processes [8]. Specifically, endothelial expression of von Willebrand factor ( $\mathrm{vWF}$ ), nitric oxide, prostanoids, endothelin-1, and thrombomodulin are altered with aging, generally leading to a procoagulatory state. Such endothelial changes are thought to be important in the development of venous thrombosis in the elderly [9]. Further, an overproduction of reactive oxygen species in red blood cells in the elderly could be involved in the etiology of arterial thrombosis [10]. Finally, it is well established that plasma concentrations of several coagulation factors (F) increase with age, especially evident for fibrinogen, FV, FVII, FVIII, FIX, and FXI (Table 1) $[7 \bullet, 11]$. Recently, the elevation of FVIII, FIX, and FXI have been specifically associated with the increased risk
Table 1 Hemostatic changes with aging

\begin{tabular}{lll}
\hline & Change & Estimated magnitude per decade \\
\hline Procoagulants & & \\
Fibrinogen (FI) & $\uparrow$ & $0.8-1 \mathrm{~g} / \uparrow \uparrow$ \\
FV & $\uparrow$ & $5-10 \%$ \\
FVIII & $\uparrow$ & $5-10 \%$ \\
FX & $(\uparrow)-\uparrow$ & $0-5 \%$ \\
FXIII & $(\uparrow)-\uparrow$ & $0-5 \%$ \\
vWF & $\uparrow$ & $10-15 \%$ \\
Anticoagulants & & \\
Protein C/S & $\uparrow$ & $\mathrm{NA}$ \\
Antithrombin & $\uparrow($ women)/ $\downarrow$ (men) & $+10 \%$ (women)/-10\% (men) \\
Markers of thrombin generation & & $10-20 \%$ \\
D-dimer & $\uparrow \uparrow$ & \\
Platelets & $\leftrightarrow$ & $\mathrm{NA}$ \\
Platelet count & $\uparrow$ & \\
Aggregation to ADP or collagen & $\leftrightarrow$ & \\
\hline
\end{tabular}

$A D P$ adenosine-di-phosphate, $N A$ not available, $\uparrow$ increased, $\downarrow$ decreased, $\leftrightarrow$ unchanged. Table adapted after $[5,12,13,80]$ 
of venous thromboembolism in the elderly [7•]. Some of these coagulation factors (i.e., fibrinogen or FVIII) act as acute phase proteins, and their elevated levels can be attributed to a low-grade chronic inflammatory state in elderly patients [6]. Regarding age-associated changes of other coagulation factors and endogenous anticoagulants including protein $\mathrm{C}$, protein $\mathrm{S}$, and antithrombin, data from different studies are conflicting and inconclusive [12,13]. Procoagulant tendency is further aggravated by reduced resolution of formed clots due to decreased fibrinolysis $[6,14]$. Finally, increased platelet reactivity has been suspected in the elderly [15]. Despite no evident changes in platelet count, it seems that lower blood concentrations of platelet agonists including adenosine-di-phosphate (ADP) and collagen are necessary to reach similar levels of platelet aggregation in the elderly compared to younger people. This increased in vitro sensitivity to platelet aggregators suggests increased platelet reactivity [5, 13]. In addition, platelet activation might be stronger in the elderly due to elevated vWF level [16]. This can be attributed to increased endothelial expression as well as decreased degradation of vWF due to age-associated reduced activity of the ADAMTS-13, an enzyme that cleaves vWF multimers.

\section{Laboratory Coagulation Testing}

Aging has been associated with different responses to various laboratory coagulation tests. While it is obvious to use age-dependent coagulation parameters in pediatric patients [17], it is less clear whether adapted normal ranges should be used in elderly and very old patients compared to adults aged $<65$ years. Increased levels of D-dimer are physiological in the elderly due to ongoing clot formation, and activated partial thromboplastin time (aPTT) is usually shortened [6] due to increased levels of various coagulation factors. These observations might complicate the correct interpretation of the results from most conventional coagulation tests in elderly patients $[18,19]$. Recent literature suggests that it might be advisable to establish and use age-adapted normal ranges in elderly patients [6]. In agreement with findings in conventional coagulation testing, viscoelastic testing might appear "hypercoagulant" because of high levels of fibrinogen and lower hematocrit due to chronic inflammation [20,21].

Of note, most conventional coagulation tests including prothrombin time (PT) and aPTT as well as viscoelastic coagulation tests preferably detect hypocoagulability, but they might be less sensitive in detecting hypercoagulability [20]. Further, most coagulation tests have demonstrated limited ability in predicting perioperative bleeding in different surgical settings. These tests should be primarily used for decision making in bleeding patients and not for screening the bleeding risk in unselected patients before surgery [22].

\section{Thromboembolic Risk in the Elderly}

The interaction of multimorbidity, procoagulant changes, and chronic low-grade inflammatory processes, which are all evident in the elderly population, increases their thromboembolic risk [5, 12, 23]. The frequency of arterial thromboembolic events including myocardial infarction and stroke is significantly elevated with aging [5]. About $80 \%$ of all fatal myocardial infarctions occur in patients aged $>70$ years [5]. Likewise, the risk of stroke increases with aging [24]. The higher prevalence of atrial fibrillation (AF) in the elderly population, in combination with age-associated hypercoagulability, significantly contributes to increased stroke risk. The importance of age as a major risk factor for stroke is well depicted in the $\mathrm{CHA}_{2} \mathrm{DS}_{2}$-VASc score, in which age $>75$ years is counted as two points [25].

Potentially of even higher interest, the incidence of venous thromboembolism (VTE) greatly increases in the elderly population ( $>75$ years) by about factor 6 (for pulmonary embolism) to 10 (for deep venous thrombosis) compared to younger people ( $<40$ years) [5]. In fact, the majority of all VTEs occur in patients aged $>70$ years [26-28]. Recurrent and fatal VTE are a major clinical concern in the elderly $[3 \bullet, 15]$. Table 2 provides a list of important comorbidities commonly associated with VTE in the elderly patients.

\section{Aging and Acquired Bleeding Disorders}

Given the increased thromboembolic risk and comorbidities associated with aging, the prescription of potent oral anticoagulants, including vitamin $\mathrm{K}$ antagonists (VKA) and direct-acting oral anticoagulants (DOAC), to the elderly is common. However, the administration of oral anticoagulants

Table 2 Comorbidities commonly associated with aging that increases thromboembolic risk

Congestive heart failure

Chronic obstructive pulmonary disease

Arteriosclerosis

Diabetes mellitus

Arterial hypertension

Atrial fibrillation

Malignancies (lymphoma, metastatic cancer)

Obesity

Sedentary lifestyle

Chronic venous insufficiency 
might increase the risk of bleeding complications in the elderly, resulting in a negative clinical benefit. In fact, the increased bleeding tendency in elderly patients should not be neglected [29]. Higher age is a risk factor for major bleeding as represented in the HAS-BLED score [25].

Most common indications for antithrombotic therapy in the elderly are $\mathrm{AF}$ and arteriosclerosis, conditions that regularly lead to intensive antithrombotic therapy with

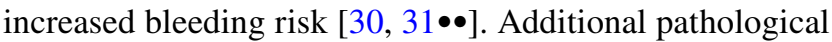
mechanisms in age-related acquired bleeding tendencies include vascular wall alterations, impaired renal function potentially leading to functional platelet disorders, and, more rarely, acquired hemophilia A and von Willebrand disease. The combination of aortic valve stenosis and gastrointestinal bleeding (Heyde's syndrome) is typically associated with aging and is thought to be related to increased rate of cleavage of high molecular weight vWF multimers. Finally, malnutrition is common in elderly, and vitamin $\mathrm{K}$ deficiency might lead to reduced levels of vitamin K-dependent coagulation factors, particularly FVII [32].

\section{Drug-Induced Bleeding Disorders}

The frequent treatment with oral anticoagulation, antiplatelet therapy, non-steroidal anti-inflammatory drugs (NSAID), and aspirin in the elderly might essentially be causative for most bleeding events. Anticoagulant therapy in elderly patients is challenging. Multiple comorbidities as well as physiological and pathological changes that occur with aging [28] render patients aged $>65$ years more vulnerable to the inherent bleeding risk of anticoagulants. Specific age-associated changes in pharmacokinetics and pharmacodynamics have been described [33]. A relative increase in body fat due to a loss of lean body mass and reduced total body water modify distribution volume of lipid soluble drugs and drug kinetics [33]. Reduced renal and hepatic clearance leads to a prolonged elimination half-time of anticoagulant drugs [33]. Pharmacodynamic changes include an increased sensitivity to anticoagulants [34]. Together, these changes are responsible for more common side effects of anticoagulant drugs in the elderly and for an increased bleeding risk in anticoagulated patients aged $>65$ years. However, withholding oral anticoagulation to avoid bleeding complications might not be justifiable in most elderly patients, as the absolute benefit

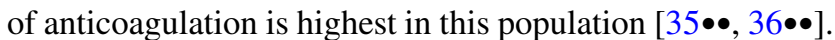
It has been estimated that oral anticoagulation is underu-

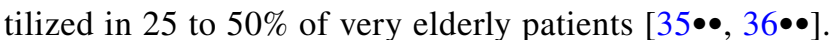
To account for pharmacokinetic and pharmacodynamic changes, lower initiation and maintenance doses might be indicated in most administered anticoagulants [37]. Of note, the higher trough concentrations of DOACs have been described in the very elderly despite dose reduction [38•]. Regular laboratory monitoring to evaluate safe drug levels and adequate efficacy might be indicated in the elderly to allow safe administration of DOACs [28]. Further, repeated assessments of the risks vs. benefits of anticoagulation and the correction and minimization of adjustable factors for increased bleeding in elderly patients while on anticoagulant therapy is strongly recommended [25, 30, 39].

For many years, the mainstay of oral anticoagulation has been VKA therapy. Recently, anticoagulation with DOACs has been associated with an increased clinical benefit in elderly patients with AF as compared to VKAs [24, 40•]. In addition, the absolute risk reduction for both ischemic and bleeding events was more pronounced with DOACs $[24,40 \bullet]$. Elderly patient might especially benefit from a reduction of bleeding events with DOACs. Subgroup analyses from the ENGAGE-AF TIMI 48 trial [41] and from the ARISTOTLE trial [42] in patients at risk of falling found DOACs to be associated with a larger absolute reduction in severe bleeding events and mortality compared to VKAs. Consistently, the risk of subdural bleeding and intracranial hemorrhage was lower in patients treated with DOACs as compared to VKAs [24]. In agreement, a recent systematic review of observational studies and RCTs found that DOACs are generally safer than VKAs in elderly AF patients, albeit that dabigatran users had a $48 \%$ increase in risk for gastro-

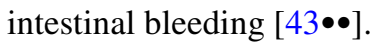

In patients with parenteral anticoagulation, those aged $>72$ years had a lower incidence of bleeding events when treated with low-molecular weight heparins (LMWH) compared to those initially treated with unfractionated heparin (UFH) for VTE [44]. Standard heparin doses commonly resulted in increased levels, prolonged aPTT, and higher anti-Xa levels in the elderly [44-46]. Therefore, lower, weight-adjusted doses of UFH might be recommended in order to avoid over-anticoagulation. In addition, tight control is necessary when therapeutic doses of heparin are administered. For initial therapy of VTE, an intravenous bolus of 80 units $/ \mathrm{kg}$ followed by about 18 units $/ \mathrm{kg} / \mathrm{h}$ is suggested $[44,45]$. On the other hand, the use of LMWH might raise concerns in the elderly, as LMWH effect is potentially prolonged in people with impaired renal function [29]. At therapeutic doses, even mild renal impairment might lead to LMWH accumulation. Nevertheless, there is lack of evidence for a clear cut-off value of creatinine clearance that would prohibit the administration of LMWH [44].

\section{Polypharmacy}

While prescribing oral anticoagulation, the physician must account for possible comorbidities and the impact of polypharmacy in the elderly that might result in considerable variability of drug effects $[47,48]$. A post-hoc analysis of the ARISTOTLE trial showed a significant increase of mortality, thromboembolic events, and bleeding complications with the 
number of concomitant drugs in patients under therapy with either apixaban or warfarin [49]. However, the relative risk reduction of stroke or thromboembolic events by apixaban was not influenced by the number of concomitant drugs. The study concluded that apixaban was more effective and safer than VKAs in patients with AF and polypharmacy [49].

Drug interactions might not only affect the potential drug efficacy but also side effects. In patients under anticoagulant therapy, the bleeding risk was massively increased by the concomitant therapy with platelet inhibitors or NSAIDs

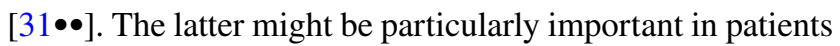
with underlying gastrointestinal lesions and ulcers. Further, selective serotonin reuptake inhibitors, particularly escitalopram, are associated with increased risk of major bleeding when combined with oral anticoagulants [50].

\section{Renal Impairment}

Aging is associated with reduced renal function and drug elimination. In addition, renal impairment affects drug binding to plasma proteins, volume of distribution, and thereby also the non-renal clearance of many drugs. Such interactions might lead to either toxicity or insufficient treatment with too high or too low drug levels, respectively [51]. Patients with chronic kidney disease (CKD) or impaired renal function who are treated with DOACs or VKAs are known to have an increased bleeding risk. The effect of impaired renal function is also reflected in different bleeding scores (e.g., HAS-BLED score) [25]. CKD might not only impair platelet function, but it is also a strong risk factor for patients to be over-anticoagulated with VKA, again increasing their bleeding risk [52]. In addition, all DOACs undergo renal elimination, at least in part. For dabigatran, renal elimination might account for up to $80 \%$ of drug clearance [53]. Regular monitoring of renal function is, therefore, of great importance in patients receiving DOACs. However, a 2017 meta-analysis evaluating RCTs comparing VKA and DOAC therapy found the latter to be associated with a lower risk of stroke and major bleeding. In patients with renal insufficiency DOACs might offer a larger net clinical benefit than VKAs [54]. Further, patients treated with DOACs showed a lower deterioration of the glomerular filtration rate over time compared to those treated with VKAs [54]. Whether these data can be directly transferred to elderly patients with limited renal impairment is not clear.

\section{The Anticoagulated Elderly Patient in the Perioperative Setting}

Older age increases the risk of multiple postoperative complications including thromboembolic events. Surgical procedures are accompanied by an intrinsic thromboembolic risk related to direct vascular damage, tissue trauma, and inflammation leading to activation of the coagulation pathways. Intraoperative positioning and postoperative immobilization increase the risk of blood stasis. For adequate estimation of perioperative thromboembolic risk, the type and extensiveness of surgery, the perioperative management, and the patient's basal conditions and comorbidities must be respected $[55,56 \bullet \bullet$.

Patient age is associated with increased risk for perioperative stroke and myocardial infarction. A previous study reported an odds ratio (OR) of 1.43 (95\% confidence interval 1.35-1.51) in the risk for perioperative stroke for each 10 -year increase in age [57]. This resulted in a two- to tentimes increased risk for perioperative stroke in octogenarians when compared to younger populations [58]. Similarly, the risk of perioperative myocardial injury/infarctions was found to increase slightly with age [59]. Further, the postoperative risk of VTE increases with age. In the recent European Society of Anaesthesiology (ESA) guidelines on perioperative thromboembolism prophylaxis in elderly patients undergoing surgery [56••], age $>70$ years was identified as clear risk factor for perioperative VTE based on several systematic reviews and large cohort studies [60-62].

However, it is important to acknowledge that individual risk for developing perioperative thromboembolism is a combination of age plus additional VTE risk factors such as obesity, a history of thromboembolic events, AF, artificial heart valves, coronary stents, cancer, lymphoma, and renal impairment [63-65]. For example, a large cohort study in more than 75,000 postoperative patients found a twofold increased for VTE in patients with renal insufficiency, leading to a significantly increased 30-day mortality [66]. It has been questioned whether age itself is an independent risk factor for postoperative thromboembolic events [56••, 67]. Rather, age might be a proxy for conditions such as frailty, multimorbidity, immobility, and low-grade coagulation activation leading to increased incidence of postoperative thromboembolic events.

\section{Prevention}

Older patients require a thoughtful preoperative geriatric assessment that should also include the use of cardiovascular risk assessment, estimation of functional capacity, and surgical risk calculators [68]. Ideally, preoperative evaluation leads to pre-habilitation, adapted anesthesia plans, minimally invasive surgical procedures, and programs for early recovery after surgery $[2,68]$. Risk factors for arterial and venous thromboembolic complications should be identified. Some common risk factors in the elderly, such as anemia or malnutrition, could potentially be corrected before elective surgery, thereby reducing adverse outcomes. Perioperative hypothermia might contribute to bleeding complications. Early application of warming systems is recommended in 
the elderly patient [69]. Although it is commonly assumed that the geriatric patient should not become hypotensive during the perioperative period to prevent stroke and myocardial ischemia [70•], there is no consensus on the most appropriate targets of perioperative blood pressure or how to achieve them. To date, there have been no RCTs showing a superiority of keeping blood pressure above a defined threshold in the perioperative period. A common practice is to maintain mean or systolic blood pressure within $20 \%$ of baseline [58]. Similarly, the use of regional or combined anesthesia rather than general anesthesia alone might reduce thromboembolic events, but the evidence is limited [71].

Perioperative thromboembolism prophylaxis must be considered in most elderly patients due to an increased inherent risk of thromboembolic complications. With respect to arterial thrombosis, timely stopping of anticoagulants and antiplatelet drugs seems important. Aspirin might be continued during the perioperative period in most patients. Aggressive bridging with LMWH or UFH should be avoided in patients with low $\mathrm{CHA}_{2} \mathrm{DS}_{2}$-VASc scores treated with VKA or DOACs. The latter has been associated with increased intraoperative and postoperative bleeding events without reducing thromboembolic complications [72•, 73]. Given the common renal and hepatic impairment with aging, polypharmacy, and the generally higher DOAC levels in the elderly, prolonged preoperative stopping intervals and laboratory drug monitoring should be considered [28, 74]. Postoperative VTE prophylaxis might be more difficult to implement in the older vs. younger patients. The recent ESA guidelines, therefore, recommended early mobilization in addition to careful prescription of postoperative VTE prophylaxis (grade 1C) [56••]. Additional multi-faceted interventions including pneumatic compression devices should be considered (grade 1C) [56••]. However, these guidelines did not recommend specific timing and dosing of pharmacological VTE prophylaxis in the elderly as compared to the

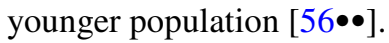

Despite intensive VTE prophylaxis, a recent retrospective study in nearly 570 patients with traumatic femoral and pelvic fractures found a consistently high incidence of postoperative thromboembolic events [75]. The authors concluded that there might be a window of opportunity by optimizing perioperative blood transfusion management, preoperative lung disease, hypoproteinemia, anemia, and potentially the inflammatory state associated with aging [75].

\section{Treatment of Thromboembolic Complications}

Early diagnosis including specific screening for perioperative thromboembolic events is thought to be the key to improving outcome after arterial and venous thromboembolic events. Systematic perioperative troponin screening for perioperative myocardial infarction has been shown to potentially reduce long-term mortality [76]. Further, the early and prompt cardiac workup including cardiac imaging and therapy of perioperative myocardial infarction might potentially improve survival, but the best strategy remains unclear. Similarly, therapy for perioperative stroke is poorly defined but increased awareness and early detection should result in improved outcomes [58].

Anticoagulation should start as soon as possible when deep venous thrombosis is diagnosed or suspected. Treatment options include subcutaneous LMWH, subcutaneous fondaparinux, or intravenous UFH. LMWH should be preferred in the elderly, as it is associated with a lower bleeding risk and less heparin-induced thrombocytopenia [77]. When administering LMWH (or fondaparinux), special attention should be paid to regularly impaired renal function to avoid excessive anticoagulation and increased bleeding risk.

In patients with postoperative pulmonary embolism, hemodynamic and respiratory support is of vital importance. Anticoagulation reduces mortality and should be administered in all elderly patients without contraindications [78]. In case of hemodynamic instability due to acute right ventricular failure, thrombolysis with streptokinase might be considered in younger as well as in elderly patients. A recent study showed a reduced mortality with acceptable bleeding complications in patients $>65$ years with pulmonary embo$\operatorname{lism}[79 \bullet]$.

\section{Conclusions}

Age-related changes in the hemostatic system in combination with frailty, reduced mobility, and age-associated comorbidities place the elderly patient at increased risk for thromboembolic events. Thereby, elderly people are especially increased risk for deep venous thrombosis and thromboembolism associated with AF. Anticoagulation should not be withheld despite an increased risk of adverse side effects and hemorrhagic complications due to changes in pharmacokinetics and pharmacodynamics associated with aging. Adapted dosing regimens and intensified drug monitoring might be considered.

In the perioperative setting, specific geriatric workup and preoperative optimization of risk factors potentially improve the outcome of the elderly surgical patients. Several strategies to prevent postoperative thromboembolic events have been suggested but evidence is limited. Early postoperative anticoagulation and monitoring of potential thromboembolic complications is recommended.

Acknowledgements The authors thank Allison Dwileski, B.S., Clinic for Anaesthesiology, Intermediate Care, Prehospital Emergency Medicina, and Pain Therapy, University Hospital Basel, Switzerland, for editorial assistance. 
Funding Open access funding provided by University of Basel.

\section{Declarations}

Conflict of interest There is no conflict of interest to declare.

Open Access This article is licensed under a Creative Commons Attribution 4.0 International License, which permits use, sharing, adaptation, distribution and reproduction in any medium or format, as long as you give appropriate credit to the original author(s) and the source, provide a link to the Creative Commons licence, and indicate if changes were made. The images or other third party material in this article are included in the article's Creative Commons licence, unless indicated otherwise in a credit line to the material. If material is not included in the article's Creative Commons licence and your intended use is not permitted by statutory regulation or exceeds the permitted use, you will need to obtain permission directly from the copyright holder. To view a copy of this licence, visit http://creativecommons.org/licenses/by/4.0/.

\section{References}

Papers of particular interest, published recently, have been highlighted as:

- Of importance

$\bullet$ Of major importance

1. United Nations, Department of Economic and Social Affairs, Population Division. World population ageing 2019: Highlights. https://www.un.org/en/development/desa/population/ publications/pdf/ageing/WorldPopulationAgeing2019-Report. pdf (accessed May 15; 2021). 2019

2. Etzioni DA, Liu JH, Maggard MA, Ko CY. The aging population and its impact on the surgery workforce. Ann Surg. 2003;238:170-7.

3. Tzoran I, Hoffman R, Monreal M. Hemostasis and thrombosis in the oldest old. Semin Thromb Hemost. 2018;44:624-31 Recent and informative review on age-associated changes in hemostasis.

4. Mari D, Ogliari G, Castaldi D, Vitale G, Bollini EM, Lio D. Hemostasis and ageing. Immun Ageing. 2008;5:12.

5. Wilkerson WR, Sane DC. Aging and thrombosis. Semin Thromb Hemost. 2002;28:555-68.

6. Favaloro EJ, Franchini M, Lippi G. Aging hemostasis: changes to laboratory markers of hemostasis as we age - a narrative review. Semin Thromb Hemost. 2014;40:621-33.

7. Wang H, Rosendaal FR, Cushman M, van Hylckama VA. Procoagulant factor levels and risk of venous thrombosis in the elderly. J Thromb Haemost. 2021;19:186-93 This cohort study evaluated specific risk of elevated coagulation factors associated with venous thrombosis in the elderly.

8. Bolliger D, Gorlinger K, Tanaka KA. Pathophysiology and treatment of coagulopathy in massive hemorrhage and hemodilution. Anesthesiology. 2010;113:1205-19.

9. Sepulveda C, Palomo I, Fuentes E. Mechanisms of endothelial dysfunction during aging: predisposition to thrombosis. Mech Ageing Dev. 2017;164:91-9.

10. Wang Q, Zennadi R. Oxidative stress and thrombosis during aging: the roles of oxidative stress in RBCs in venous thrombosis. Int J Mol Sci. 2020;21:4259.

11. Hager K, Setzer J, Vogl T, Voit J, Platt D. Blood coagulation factors in the elderly. Arch Gerontol Geriatr. 1989;9:277-82.
12. Bucciarelli P, Mannucci PM. The hemostatic system through aging and menopause. Climacteric. 2009;12(Suppl 1):47-51.

13. Franchini M. Hemostasis and aging. Crit Rev Oncol Hematol. 2006;60:144-51.

14. Ochi A, Adachi T, Inokuchi K, Ogawa K, Nakamura Y, Chiba Y, et al. Effects of aging on the coagulation fibrinolytic system in outpatients of the cardiovascular department. Circ J. 2016;80:2133-40.

15. Sepulveda C, Palomo I, Fuentes E. Primary and secondary haemostasis changes related to aging. Mech Ageing Dev. 2015;150:46-54.

16. Jalaer I, Tsakiris DA, Solecka-Witulska BA, Kannicht C. The role of von Willebrand factor in primary haemostasis under conditions of haemodilution. Thromb Res. 2017;157:142-6.

17. Toulon P, Berruyer M, Brionne-Francois M, Grand F, Lasne D, Telion $\mathrm{C}$, et al. Age dependency for coagulation parameters in paediatric populations results of a multicentre study aimed at defining the age-specific reference ranges. Thromb Haemost. 2016;116:9-16.

18. Favaloro EJ, Soltani S, McDonald J, Grezchnik E, Easton L. Cross-laboratory audit of normal reference ranges and assessment of ABO blood group, gender and age on detected levels of plasma coagulation factors. Blood Coagul Fibrinolysis. 2005; 16:597-605.

19. Lippi G, Favaloro EJ, Cervellin G. A review of the value of D-dimer testing for prediction of recurrent venous thromboembolism with increasing age. Semin Thromb Hemost. 2014;40:634-9.

20. Bolliger D, Seeberger MD, Tanaka KA. Principles and practice of thromboelastography in clinical coagulation management and transfusion practice. Transfus Med Rev. 2012;26:1-13.

21. Ogawa S, Szlam F, Bolliger D, Nishimura T, Chen EP, Tanaka KA. The impact of hematocrit on fibrin clot formation assessed by rotational thromboelastometry. Anesth Analg. 2012;115:16-21.

22. Larsen JB, Hvas AM. Predictive value of whole blood and plasma coagulation tests for intra- and postoperative bleeding risk: a systematic review. Semin Thromb Hemost. 2017;43:772-805.

23. Kop WJ, Gottdiener JS, Tangen CM, Fried LP, McBurnie MA, Walston J, et al. Inflammation and coagulation factors in persons $>65$ years of age with symptoms of depression but without evidence of myocardial ischemia. Am J Cardiol. 2002;89:419-24.

24. Bauersachs RM, Herold J. Oral anticoagulation in the elderly and frail. Hamostaseologie. 2020;40:74-83.

25. Kato ET, Giugliano RP, Ruff CT, Koretsune Y, Yamashita $\mathrm{T}$, Kiss RG, et al. Efficacy and safety of edoxaban in elderly patients with atrial fibrillation in the ENGAGE AF-TIMI 48 trial. J Am Heart Assoc 2016;5

26. Cook LM, Kahn SR, Goodwin J, Kovacs MJ. Frequency of renal impairment, advanced age, obesity and cancer in venous thromboembolism patients in clinical practice. J Thromb Haemost. 2007;5:937-41.

27. Engbers MJ, van Hylckama VA, Rosendaal FR. Venous thrombosis in the elderly: incidence, risk factors and risk groups. $\mathrm{J}$ Thromb Haemost. 2010;8:2105-12.

28. Ali MS, Czarnecka-Kujawa K. Venous thromboembolism in the elderly. Curr Geri Rep. 2016;5:132-9.

29. Kruse-Jarres R. Acquired bleeding disorders in the elderly. Hematology Am Soc Hematol Educ Program. 2015;2015:231-6.

30. Bolliger D, Fassl J, Erdoes G. How to manage the perioperative patient on combined anticoagulant and antiplatelet therapy: comments on the 2020 ACC Consensus Decision Pathway. J Cardiothorac Vasc Anesth. 2021;35:1561-4.

31.• Kumbhani DJ, Cannon CP, Beavers CJ, Bhatt DL, Cuker A, Gluckman TJ, et al. 2020 ACC Expert Consensus Decision 
Pathway for anticoagulant and antiplatelet therapy in patients with atrial fibrillation or venous thromboembolism undergoing percutaneous coronary intervention or with atherosclerotic cardiovascular disease: a report of the American College of Cardiology Solution Set Oversight Committee. J Am Coll Cardiol. 2021;77:629-58 undefined. Inthis concensus paper, experts recommend optimal procedures in patients oncombined anticoagulant and antiplatelet therapy based on best evidence

32. Woehrle D, Martinez M. Bolliger D [Hereditary heterozygous factor VII deficiency in patients undergoing surgery : clinical relevance]. Anaesthesist. 2016;65:746-54.

33. Turnheim K. Drug therapy in the elderly. Exp Gerontol. 2004;39:1731-8.

34. Mangoni AA, Jackson SH. Age-related changes in pharmacokinetics and pharmacodynamics: basic principles and practical applications. Br J Clin Pharmacol. 2004;57:6-14.

35.• Patti G, Lucerna M, Pecen L, Siller-Matula JM, Cavallari I, Kirchhof P, et al. Thromboembolic risk, bleeding outcomes and effect of different antithrombotic strategies in very elderly patients with atrial fibrillation: a sub-analysis from the PREFER in AF (PREvention oF Thromboembolic EventsEuropean Registry in Atrial Fibrillation). J Am Heart Assoc 2017;6:e05657. This study support the use of anticoagulant therapy in most very elderly patients with atrial fibrillation despite common concerns of bleeding

36.• Fohtung RB, Novak E, Rich MW. Effect of new oral anticoagulants on prescribing practices for atrial fibrillation in older adults. J Am Geriatr Soc. 2017;65:2405-12 This important cohort study showed that oral anticoagulants should not be withhold in the elderly.

37. Garcia D, Regan S, Crowther M, Hughes RA, Hylek EM. Warfarin maintenance dosing patterns in clinical practice: implications for safer anticoagulation in the elderly population. Chest. 2005; 127:2049-56.

38. Gommans E, Grouls RJE, Kerkhof D, Houterman S, Simmers $\mathrm{T}$, Van der Linden C. Dabigatran trough concentrations in very elderly patients. Eur J Hosp Pharm 2020; epub ahead. This study showed that DOAC levels tend to be elevated in the elderly despite adapted dose regimen

39. Lopez-Jimenez L, Montero M, Gonzalez-Fajardo JA, Arcelus JI, Suarez C, Lobo JL, et al. Venous thromboembolism in very elderly patients: findings from a prospective registry (RIETE). Haematologica. 2006;91:1046-51.

40. Patti G, Pecen L, Lucerna M, Huber K, Rohla M, Renda G, et al. Net clinical benefit of non-vitamin $\mathrm{K}$ antagonist vs vitamin $\mathrm{K}$ antagonist anticoagulants in elderly patients with atrial fibrillation. Am J Med 2019;132:749-57 e5. The use of DOAC instead of VKA might be associated with clincial benefits in the elderly patients. Both bleeding and thromboembolic events were lower in the DOAC groupd

41. Steffel J, Giugliano RP, Braunwald E, Murphy SA, Mercuri M, Choi $\mathrm{Y}$, et al. Edoxaban versus warfarin in atrial fibrillation patients at risk of falling: ENGAGE AF-TIMI 48 analysis. J Am Coll Cardiol. 2016;68:1169-78.

42. Rao MP, Vinereanu D, Wojdyla DM, Alexander JH, Atar D, Hylek EM, et al. Clinical outcomes and history of fall in patients with atrial fibrillation treated with oral anticoagulation: Insights from the ARISTOTLE trial. Am J Med. 2018;131:269-75 e2.

43.• Shen NN, Wu Y, Wang N, Kong LC, Zhang C, Wang JL, et al. Direct oral anticoagulants vs. vitamin-K antagonists in the elderly with atrial fibrillation: a systematic review comparing benefits and harms between observational studies and randomized controlled trials. Front Cardiovasc Med 2020;7:132. In this systematic review, the authors showed that the use of
DOACs is associated with a clinical benefit in the elderly as compared to the use of VKAs

44. Dorobantu M, Bogdan S. Unfractionated heparin or lowmolecular-weight heparin in the elderly. Int $\mathrm{J}$ Cardiol. 2016;222:1084-90.

45. Kearon C, Kahn SR, Agnelli G, Goldhaber S, Raskob GE, Comerota AJ. Antithrombotic therapy for venous thromboembolic disease: American College of Chest Physicians Evidence-Based Clinical Practice Guidelines (8th Edition). Chest 2008; 133:454S-545S.

46. Lemke A, Kohs J, Weber L. Evaluating anticoagulation sensitivity among elderly patients managed with an institution's heparin protocol using initial anti-factor Xa levels. Am J Health Syst Pharm. 2020;77:S13-8.

47. Chang SH, Chou IJ, Yeh YH, Chiou MJ, Wen MS, Kuo CT, et al. Association between use of non-vitamin $\mathrm{K}$ oral anticoagulants with and without concurrent medications and risk of major bleeding in nonvalvular atrial fibrillation. JAMA. 2017;318:1250-9.

48. Barben J, Menu D, Rosay C, Vovelle J, Mihai AM, Nuss V, et al. The prescription of direct oral anticoagulants in the elderly: an observational study of 19798 Ambulatory subjects. Int J Clin Pract. 2020;74:e13420.

49. Jaspers Focks J, Brouwer MA, Wojdyla DM, Thomas L, Lopes $\mathrm{RD}$, Washam JB, et al. Polypharmacy and effects of apixaban versus warfarin in patients with atrial fibrillation: post hoc analysis of the ARISTOTLE trial. BMJ. 2016;353:i2868.

50. Samuel NG, Seifert CF. Risk of bleeding in patients on full-dose enoxaparin with venous thromboembolism and selective serotonin reuptake inhibitors. Ann Pharmacother. 2017;51:226-31.

51. Dreisbach AW, Lertora JJ. The effect of chronic renal failure on drug metabolism and transport. Expert Opin Drug Metab Toxicol. 2008;4:1065-74.

52. Limdi NA, Beasley TM, Baird MF, Goldstein JA, McGwin G, Arnett DK, et al. Kidney function influences warfarin responsiveness and hemorrhagic complications. J Am Soc Nephrol. 2009;20:912-21.

53. Bauersachs RM. Use of anticoagulants in elderly patients. Thromb Res. 2012;129:107-15.

54. Zou R, Tao J, Shi W, Yang M, Li H, Lin X, et al. Meta-analysis of safety and efficacy for direct oral anticoagulation treatment of non-valvular atrial fibrillation in relation to renal function. Thromb Res. 2017;160:41-50.

55. Douketis JD, Spyropoulos AC, Spencer FA, Mayr M, Jaffer AK, Eckman MH, et al. Perioperative management of antithrombotic therapy: antithrombotic therapy and prevention of thrombosis, 9th ed: American College of Chest Physicians Evidence-Based Clinical Practice Guidelines. Chest. 2012;141:e326S - e350.

56.• Kozek-Langenecker S, Fenger-Eriksen C, Thienpont E, Barauskas G, Force EVGT. European guidelines on perioperative venous thromboembolism prophylaxis: Surgery in the elderly. Eur J Anaesthesiol. 2018;35:116-22 Recent European guidelines on the best evidence in postoperative venous thromboembolism prophylaxis in the elderly.

57. Bateman BT, Schumacher HC, Wang S, Shaefi S, Berman MF. Perioperative acute ischemic stroke in noncardiac and nonvascular surgery: incidence, risk factors, and outcomes. Anesthesiology. 2009;110:231-8.

58. $\mathrm{Ng} \mathrm{JL}$, Chan MT, Gelb AW. Perioperative stroke in noncardiac, nonneurosurgical surgery. Anesthesiology. 2011;115:879-90.

59. Gualandro DM, Puelacher C, Lurati Buse G, Glarner N, Cardozo FA, Vogt R, et al. Incidence and outcomes of perioperative myocardial infarction/injury diagnosed by high-sensitivity cardiac troponin I. Clin Res Cardiol 2021; epub ahead 
60. Lee E, Kang SB, Choi SI, Chun EJ, Kim MJ, Kim DW, et al. Prospective study on the incidence of postoperative venous thromboembolism in Korean patients with colorectal cancer. Cancer Res Treat. 2016;48:978-89.

61. Mauck KF, Froehling DA, Daniels PR, Dahm DL, Ashrani AA, Crusan DJ, et al. Incidence of venous thromboembolism after elective knee arthroscopic surgery: a historical cohort study. J Thromb Haemost. 2013;11:1279-86.

62. Zhang J, Chen Z, Zheng J, Breusch SJ, Tian J. Risk factors for venous thromboembolism after total hip and total knee arthroplasty: a meta-analysis. Arch Orthop Trauma Surg. 2015;135:759-72.

63. Kikura M, Takada T, Sato S. Preexisting morbidity as an independent risk factor for perioperative acute thromboembolism syndrome. Arch Surg. 2005;140:1210-7.

64. Sakon M, Maehara Y, Yoshikawa H, Akaza H. Incidence of venous thromboembolism following major abdominal surgery: a multi-center, prospective epidemiological study in Japan. J Thromb Haemost. 2006;4:581-6.

65. White RH, Zhou H, Gage BF. Effect of age on the incidence of venous thromboembolism after major surgery. J Thromb Haemost. 2004;2:1327-33.

66. Gangireddy C, Rectenwald JR, Upchurch GR, Wakefield TW, Khuri S, Henderson WG, et al. Risk factors and clinical impact of postoperative symptomatic venous thromboembolism. J Vasc Surg. 2007;45:335-41.

67. Landesberg G, Beattie WS, Mosseri M, Jaffe AS, Alpert JS. Perioperative myocardial infarction. Circulation. 2009;119:2936-44.

68. Aceto P, Antonelli Incalzi R, Bettelli G, Carron M, Chiumiento F, Corcione A, et al. Perioperative management of elderly patients (PriME): recommendations from an Italian intersociety consensus. Aging Clin Exp Res. 2020;32:1647-73.

69. Lim BG, Lee IO. Anesthetic management of geriatric patients. Korean J Anesthesiol. 2020;73:8-29.

70.• Gregory A, Stapelfeldt WH, Khanna AK, Smischney NJ, Boero IJ, Chen Q, et al. Intraoperative Hypotension is associated with adverse clinical outcomes after noncardiac surgery. Anesth Analg. 2021;132:1654-65 In this retrospective analysis including $>350,000$ patients undergoing non-cardiac surgery, intraoperative hypotension was associated with postoperative thromboembolic events and should therefore be avoided.

71. Guay J, Choi PT, Suresh S, Albert N, Kopp S, Pace NL. Neuraxial anesthesia for the prevention of postoperative mortality and major morbidity: an overview of cochrane systematic reviews. Anesth Analg. 2014;119:716-25.
72. Douketis JD, Spyropoulos AC, Duncan J, Carrier M, Le Gal G, Tafur AJ, et al. Perioperative management of patients with atrial fibrillation receiving a direct oral anticoagulant. JAMA Intern Med. 2019;179:1469-78 In this prospective cohort study, stopping DOAC 24 and 48 hours before elective minor and major sugery was safe in most patients. However, it is not fully clear whether this conclusion also applies to elderly patients, as they are known to have higher trough concentrations.

73. Douketis JD, Spyropoulos AC, Kaatz S, Becker RC, Caprini JA, Dunn AS, et al. Perioperative bridging anticoagulation in patients with atrial fibrillation. N Engl J Med. 2015;373:823-33.

74. Olotu C, Weimann A, Bahrs C, Schwenk W, Scherer M, Kiefmann R. The perioperative care of older patients. Dtsch Arztebl Int. 2019;116:63-9.

75. Wu L, Cheng B. Analysis of perioperative risk factors for deep vein thrombosis in patients with femoral and pelvic fractures. $\mathrm{J}$ Orthop Surg Res. 2020;15:597.

76. Puelacher C, Lurati Buse G, Seeberger D, Sazgary L, Marbot S, Lampart A, et al. Perioperative myocardial injury after noncardiac surgery: Incidence, mortality, and characterization. Circulation. 2018;137:1221-32.

77. Mahe I, Gouin-Thibault I, Drouet L, Simoneau G, Di Castillo H, Siguret V, et al. Elderly medical patients treated with prophylactic dosages of enoxaparin: influence of renal function on anti-Xa activity level. Drugs Aging. 2007;24:63-71.

78. Berman AR. Pulmonary embolism in the elderly. Clin Geriatr Med. 2001;17:107-30.

79.• Ipek G, Karatas MB, Onuk T, Gungor B, Yuzbas B, Keskin $\mathrm{M}$, et al. Effectiveness and safety of thrombolytic therapy in elderly patients with pulmonary embolism. J Thromb Thrombolysis. 2015;40:424-9 In this cohort study, the authors demonstrated that thrombolytic therapy should also be used in the elderly patient.

80. Hamilton PJ, Allardyce M, Ogston D, Dawson AA, Douglas AS. The effect of age upon the coagulation system. J Clin Pathol. 1974;27:980-2.

Publisher's Note Springer Nature remains neutral with regard to jurisdictional claims in published maps and institutional affiliations. 